\title{
A Switched-Capacitor Digital Capacitance Bridge
}

\author{
KENZO WATANABE, MEMBER, IEEE, AND GABOR C. TEMES, FELLOW, IEEE
}

Abstract-A switched-capacitor bridge has been developed for capacitance measurements. It consists of four arms connected between the low-impedance output and virtual ground nodes of an op-amp, and hence is insensitive to parasitic capacitances. The capacitance to be measured is first given a proportional charge. This charge is then compared successively with charges quantized by means of a programmable binary-weighted capacitor array, until a balance is reached. This digital balance operation makes it possible to accomodate an automatic calibration scheme, which affords, in conjunction with the parasitic-insensitive configuration, an accurate measurement. Error analysis has shown that a 10-bit quantization accuracy and a relative error as small as 0.1 percent are attainable when the bridge is fabricated in LSI circuit form using present MOS technologies. A prototype bridge built using discrete components has confirmed the principles of operation. Examples of measurement are also given.

\section{INTRODUCTION}

B ALANCING a bridge by nulling a meter is a fundamental procedure for measuring a capacitance using the transformer-ratio-arm or Schering bridge [1]. A measurement accuracy of 1 part $/ 10^{6}$ is attainable by this method, but the procedure is cumbersome and time consuming because of the manual balance operation involved. The four-terminal-pair method [2] has eliminated the manual operation by detecting the voltage across and the current through the impedance to be measured. The price paid for the automated measurement is the complicated signal processing necessary for calculating the impedance. In addition, this method requires a special sample holder because it is susceptible to parasitic capacitances [3] This makes it difficult to apply this method to the characterization of integrated MOS capacitors in which parasitic capacitances are inevitable.

McCreary and Sealer have proposed a novel technique to characterize binary-weighted MOS capacitor arrays [4]. Based on the voltage-divider principle, their method has facilitated the capacitor ratio measurement, but it cannot measure the absolute value of each capacitor.

In a recent publication [5], a switched-capacitor circuit has been proposed for digital multiplication. This circuit has been modified to form a capacitance bridge which allows quick measurement of the absolute value as well as the ratio of capacitors. This paper describes the bridge configuration, the principles of measurement, and calculates the expected accuracy. A prototype bridge implemented using discrete components and some examples of measurement are also presented.

Manuscript received November 28, 1983; revised March 8, 1984. This Work was supported in part by the National Science Foundation under Grant ECS-8105166.

$\mathrm{K}$. Watanabe is with the Research Institute of Electronics, Shizuoka University, Hamamatsu 432, Japan.

G. C. Temes is with the Department of Electrical Engineering, University of California, Los Angeles, CA 90024.

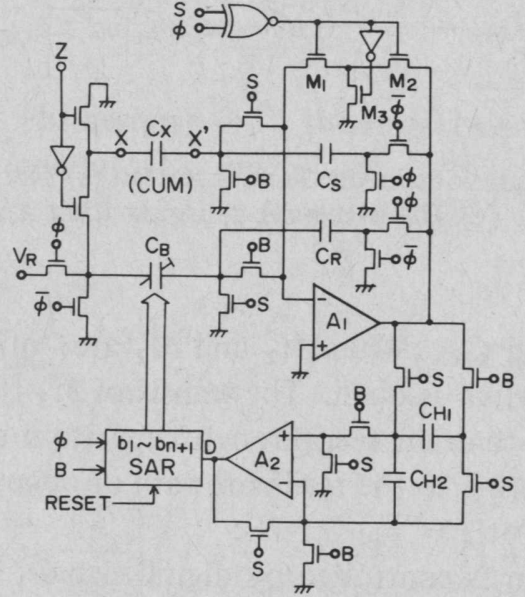

(a)

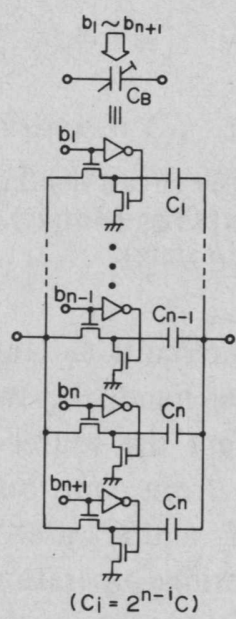

(b)

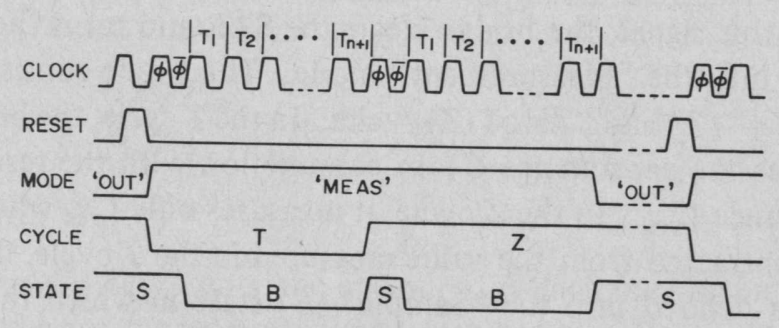

(c)

Fig. 1. (a) The schematic diagram of the capacitance bridge. CUM denotes the "Capacitor Under Measurement" and SAR the "SuccessiveApproximation Register." (b) The configuration of the programmable binary-weighted capacitor array $C_{B}$. (c) The timing sequence of the digital signals controlling the bridge operation.

\section{The Capacitance Bridge}

Fig. 1(a) shows the schematic diagram of the capacitance bridge. The capacitor $C_{X}$ under measurement (CUM) is connected between the terminals $X-X^{\prime}$. Its value is to be measured with reference to $C_{S}$. Therefore, $C_{S}$ must be a standard capacitor of known value when an absolute value measurement is desired. $C_{B}$ is a programmable binary-weighted capacitor array whose configuration is shown in Fig. 1(b). $C_{B}$ is related to the reference capacitor $C_{R}$. These four capacitors $C_{X}, C_{S}, C_{B}$, and $C_{R}$ form the four arms of the bridge. Since each arm is connected between a voltage source and the virtual ground of op-amp $A_{1}$, the parasitic capacitances to ground have no effect upon the bridge balance if op-amp $A_{1}$ is ideal. The holding capacitor $C_{H_{2}}$ corresponds to the detector arm of a conventional bridge. Controlled by the signal from op-amp $A_{2}$, the successive-approximation registers SAR programs $C_{B}$ so that the charge on $\mathrm{C}_{\mathrm{H}_{2}}$ becomes zero, thereby bringing the bridge into a balance.

The transistors $M_{1}, M_{2}$, and $M_{3}$ form a reset switch. When $M_{1}$ and $M_{2}$ are "on" and $M_{3}$ is "off," the switch is closed and 


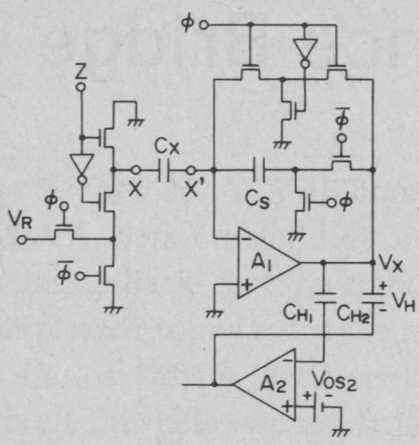

(a)

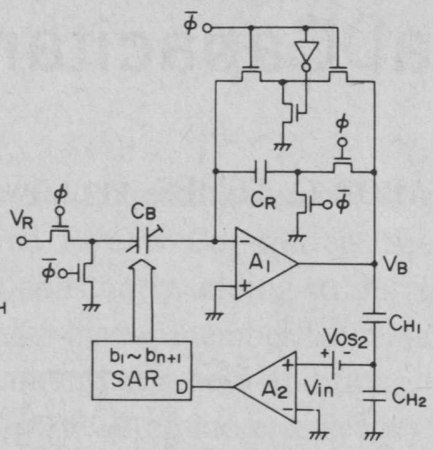

(b)
Fig. 2. The circuit configurations in the $S$ and $B$ states: (a) The offsetfree amplifier ( $S$ state). (b) The successive-approximation A/D converter ( $B$ state).

it short circuits $C_{S}$ and $C_{R}$. When $M_{1}$ and $M_{2}$ are "off," on the other hand, the switch is open. The transistor $M_{3}$ is "on" to prevent the source-to-drain feedthrough capacitors of $M_{1}$ and $M_{2}$ from contributing to the feedback path of op-amp $A_{1}$ when $M_{1}$ and $M_{2}$ are "off."

The bridge operation is controlled by digital signals, whose timing sequence is shown in Fig. 1(c). A "Reset" signal sent from an external device initiates the measurement. Upon receiving this signal, the bridge clears the SAR and turns the operation into the "Measurement" mode. This mode consists of the "Test" $(T)$ and "Zero" $(Z)$ cycles. In the $T$ cycle, the bridge measures the capacitance $C_{X}$ in conjunction with the residual capacitance $C_{\text {res }}$. In the $Z$ cycle, it measures only $C_{\text {res }}$ which is then subtracted from the value measured in the $T$ cycle. Each cycle is divided into the "Sample" $(S)$ state in which the capacitor being measured is given a proportional charge, and the "Balance" $(B)$ state during which the charge is approximately balanced with a charge quantized by means of a successiveapproximation A/D conversion. When the $B$ state in the $Z$ cycle is completed, the operation changes into the "Out" mode. The capacitance value $C_{X}$ is then available in the SAR in binary form. This mode continues until the next reset signal starts a new cycle of measurement. The operation in the $T$ cycle will be next described in detail.

\section{A. Operation in the S State of the T Cycle}

In this state, $S=1$ and $B=0$. Thus the capacitors $C_{X}$ and $C_{S}$ are connected to the inverting input terminal of op-amp $A_{1}$ to form an offset-free noninverting amplifier [6]. Since $Z=0$, the input of this circuit is the reference voltage $V_{R}$ in the $\bar{\phi}=1$ phase, as shown in Fig. 2(a). The output voltage is therefore

$$
V_{X}=\left(C_{X} / C_{S}\right) V_{R}
$$

The op-amp $A_{2}$ forms a voltage follower with a grounded input. Its output is thus its own input-referred offset voltage $V_{\mathrm{os}_{2}}$. The two holding capacitors $C_{H_{1}}$ and $C_{H_{2}}$ are connected in parallel in this state. Their top and bottom plates are driven by op-amps $A_{1}$ and $A_{2}$, respectively. Therefore, the voltages $V_{H}$ across $C_{H_{2}}$ is $V_{X}-V_{\mathrm{os}_{2}}$. This voltage represents the initial voltage of $\mathrm{C}_{\mathrm{H}_{2}}$ during the subsequent $B$ state if the circuit condition is ideal. The operation under nonideal conditions will be described in Section III.

\section{B. Operation in the B State of the T Cycle}

Since now $B=1$ and $S=0$, the bridge contains $C_{B}$ and $C_{R}$ in place of $C_{X}$ and $C_{S}$, respectively. The op-amp $A_{1}$ then forms an offset-free D/A converter. $C_{H_{1}}$ and $C_{H_{2}}$ are now connected in series, holding the voltage $V_{H}$ stored in the previous $S$ state. The op-amp $A_{2}$ now operates as a comparator. The whole circuit, including the SAR, is shown in Fig. 2(b). It forms a successive-approximation A/D converter [8]; the voltage to be converted is $V_{H}+V_{\mathrm{os}_{2}}=V_{X}$.

The A/D conversion is performed as follows. In time slot $T_{i}(i=1,2, \cdots, n+1)$, the $i$ th bit of the SAR $\left(b_{i}\right)$ is tempo. rarily set to 1 , while the previously tested $i-1$ values of $b$ are stored. The capacitance of the array $C_{B}$ is then

$$
C_{B}^{(i)}=\sum_{j=1}^{i-1} b_{j} \cdot 2^{n-j} C+2^{n-i} C
$$

Charging this capacitor to the reference voltage $V_{R}$ in the $\phi=1$ phase, the $\mathrm{D} / \mathrm{A}$ converter produces an output

$$
V_{B}=-\left(C_{B}^{(i)} / C_{R}\right) V_{R} \text {. }
$$

This tentative output value is divided by capacitors $C_{H_{1}}$ and $\mathrm{C}_{\mathrm{H}_{2}}$. The input voltage $V_{\mathrm{in}}$ of the comparator is then

$$
\begin{aligned}
V_{\text {in }} & =V_{H}+V_{\mathrm{os}_{2}}+\frac{C_{H_{1}}}{C_{H_{1}}+C_{H_{2}}} V_{B} \\
& =\left[\frac{C_{X}}{C_{S}}-\frac{C_{H_{1}}}{C_{H_{1}}+C_{H_{2}}} \cdot \frac{C_{B}^{(i)}}{C_{R}}\right] V_{R} .
\end{aligned}
$$

Depending on the polarity of $V_{\text {in }}$, the SAR keeps $b_{i}$ as 1 (if $V_{\text {in }}$ is positive) or resets it to 0 (if $V_{\text {in }}$ is negative). Repeating this process $n+1$ times makes $V_{\text {in }}$ approximately zero, so that

$$
\frac{C_{X}}{C_{S}} \simeq \frac{C_{H_{1}}}{C_{H_{1}}+C_{H_{2}}} \cdot \frac{C_{B}}{C_{R}}
$$

holds, where

$$
C_{B}=\sum_{i=1}^{n} b_{i} \cdot 2^{n-i} C+b_{n+1} C .
$$

When $C_{H_{1}}=C_{H_{2}}$ and $C_{R}=2^{n-1} C$, the SAR stores the ratio $C_{X} / C_{S}$ (i.e., the capacitance $C_{X}$ scaled by $C_{S}$ ) in binary form

$$
C_{X} / C_{S}=b_{1} \cdot 2^{-1}+b_{2} \cdot 2^{-2}+\cdots+b_{n} \cdot 2^{-n}+b_{n+1} \cdot 2^{-n}
$$

It is worth noting here that the above operation is not affected by op-amp offset voltages if the open-loop gains of opamps $A_{1}$ and $A_{2}$ are sufficiently high. Therefore, the bridge can be fabricated in a fully integrated form by MOS IC technologies.

\section{Accuracy Estimate}

The operation of the practical bridge circuit is affected by many error sources, such as mismatches in the capacitance ratio between $C_{B}$ and $C_{R}$, the offset voltages and finite gains of opamps $A_{1}$ and $A_{2}$, parasitic capacitances, and the feedthrough of the clock signals. These nonideal circuit conditions disturb the ideal balance condition, imposing a limit on the measure- 


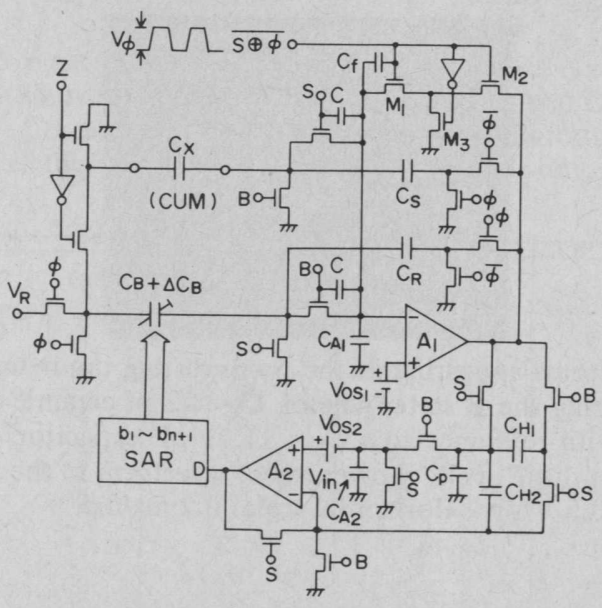

Fig. 3. The practical bridge circuit, including parasitic elements.

ment accuracy. In this section, we calculate the estimated accuracy when all of the bridge circuit (except $C_{X}$ and $C_{S}$ ) is implemented by MOS IC process.

The bridge in Fig. 1(a) contains, in fact, many parasitic capacitances including the gate-source and gate-drain feedthrough capacitances of the MOS switches. Those residual capacitances, however, which are connected to the low-impedance nodes (except to virtual grounds) have no effect on the bridge balance. Furthermore, some of the feedthrough capacitances have matched couterparts driven by a complementary clock signal, such as $C_{\epsilon}$ and $C_{\delta}$ in Fig. 3. The feedthrough charges due to these paired capacitances can be neglected in a first-order approximation because they tend to cancel each other. Extending the above considerations into the overall circuit and including the other nonideal effects, we find the practical circuit model of the bridge shown in Fig. 3. In this figure, $C_{f}$ is the feedthrough capacitance of $M_{1}, C_{p}$ is the combined top-plate stray capacitance of $C_{H_{1}}$ and $C_{H_{2}}$, and $C_{A_{1}}$ and $C_{A_{2}}$ are the input capacitances of op-amps $A_{1}$ and $A_{2}$, respectively. The mismatch between $C_{B}$ and $C_{R}$ is represented by $\Delta C_{B}$.

The output voltage of op-amp $A_{1}$ in the $\bar{\phi}=1$ phase of the $S$ state is now, assuming $Z=0$

$$
V_{X}^{\prime}=\frac{C_{X} V_{R}+C_{f} V_{\phi}+C_{\mathrm{TS}} V_{\mathrm{os}_{1}} /\left(1+A_{1}\right)}{C_{S}\left(1+C_{\mathrm{TS}} / C_{S} A_{1}\right)}
$$

where $V_{\phi}$ is the amplitude of the clock signal applied to the reset transistor $M_{1}$, and

$$
C_{\mathrm{TS}}=C_{X}+C_{S}+C_{f}+C_{\epsilon}+C_{\delta}+C_{A_{1}}
$$

is the sum of the capacitances connected to the inverting input terminal of $A_{1}$ in the $S$ state. The voltage follower $A_{2}$ produces the output voltage

$$
V_{A}^{\prime}=\frac{A_{2}}{1+A_{2}} V_{\mathrm{os}_{2}} \text {. }
$$

The voltage across the holding capacitors $C_{H_{1}}$ and $C_{H_{2}}$ is thus $V_{X}^{\prime}-V_{A}^{\prime}$, while that across $C_{p}$ is $V_{X}^{\prime}$.

When the successive-approximation A/D conversion is completed, op-amp $A_{1}$ produces the output voltage

$$
V_{B}^{\prime}=\frac{-\left(C_{B}+\Delta C_{B}\right) V_{R}+C_{f} V_{\phi}+C_{\mathrm{TB}} V_{\mathrm{os}_{1}} /\left(1+A_{1}\right)}{C_{R}\left(1+C_{\mathrm{TB}} / C_{R} A_{1}\right)} .
$$

Here

$$
C_{\mathrm{TB}}=C_{\mathrm{BT}}+C_{R}+C_{f}+C_{\epsilon}+C_{\delta}+C_{A_{1}}
$$

is the sum of the capacitances connected to the inverting input terminal of $A_{1}$ in the $B$ state, and $C_{\mathrm{BT}}=2^{n} C$ is the total capacitance of the array $C_{B}$.

The op-amp $A_{2}$ and the SAR balance the bridge so that

$$
V_{\text {in }}=\frac{2 C_{H}\left(V_{H}^{\prime}-V_{A}^{\prime}\right)+C_{p} V_{X}^{\prime}+C_{H} V_{B}^{\prime}}{2 C_{H}+C_{p}+C_{A_{2}}}+V_{\mathrm{os}_{2}}=0
$$

holds. In deriving (13), the mismatch between $C_{H_{1}}$ and $C_{H_{2}}$ is lumped into $C_{p}$ and thus $C_{H_{1}}=C_{H_{2}}=C_{H}$ is assumed without loss of generality. Substituting (8), (10), and (11) into (13), we have

$$
C_{\text {meas }}=\frac{C_{S}\left(C_{B}+\Delta C_{B}\right)}{2 C_{R}}+\epsilon C_{X}+C_{\text {res }}
$$

where

$$
\begin{aligned}
\epsilon=\left[1+\frac{C_{p}}{2 C_{H}}\right]\left[1+\frac{1}{A_{1}} \cdot \frac{C_{\mathrm{TB}}}{C_{R}}\right] /\left[1+\frac{1}{A_{1}} \cdot \frac{C_{\mathrm{TS}}}{C_{S}}\right]-1 \\
C_{\mathrm{res}}=\left[1+\epsilon+\frac{C_{S}}{2 C_{R}}\right] \frac{C_{f} V_{\phi}}{V_{R}}+\left[1+\frac{(1+\epsilon) C_{\mathrm{TS}}}{C_{S}}\right] \\
\cdot \frac{C_{S} V_{\mathrm{os}_{1}}}{\left(1+A_{1}\right) V_{R}}+\left[\frac{1}{1+A_{2}}+\frac{C_{p}+C_{A_{2}}}{2 C_{H}}\right] \frac{C_{S} V_{\mathrm{os}_{2}}}{V_{R}}
\end{aligned}
$$

$C_{B}+\Delta C_{B}$ represents the measured capacitance including the digital error caused by the mismatch between $C_{B}$ and $C_{R}$, while $\epsilon C_{X}+C_{\text {res }}$ represents the analog error due to parasitic capacitances, clock feedthrough, and the nonideal performances of op-amps $A_{1}$ and $A_{2}$. They will be discussed separately in the following.

\section{A. Digital Error}

Each capacitor in the array $C_{B}$ is assumed to be fabricated by connecting an appropriate number of MOS unit capacitors of magnitude $C$ in parallel. The error in each capacitor is assumed to be random, normally distributed, and uncorrelated. The error charge $\Delta Q_{B}$ in the array $C_{B}$ due to the capacitance error is then given by

$$
\Delta Q_{B}=\sqrt{\sum_{i=1}^{n+1}\left(b_{i} \cdot \Delta C_{i}\right)^{2}} V_{R}
$$

where $\Delta C_{i}$ is the standard deviation of each capacitor. If this error charge is smaller than half of that held in the LSB capacitor, then the $\mathrm{A} / \mathrm{D}$ conversion process is monotonic and the digital output is accurate down to its LSB. Formulating this in terms of the capacitance mismatch, we have the condition

$$
\sqrt{\sum_{i=1}^{n+1}\left(\Delta C_{i} / C_{\mathrm{BT}}\right)^{2}} \leqslant 2^{-(n+1)}
$$

Each term under the square root can be estimated by using the data presented by McCreary [7]: $\Delta C_{1} / C_{\mathrm{BT}}=0.03$ percent, $\Delta C_{2} / C_{\mathrm{BT}}=0.02$ percent, $\Delta C_{3} / C_{\mathrm{BT}}=0.015$ percent, and so 
on. Substituting these values into (18), we find that the quantization accuracy obtainable with present MOS technologies is 10 bits [8]

\section{B. Analog Error}

The analog error can be described in terms of the relative error $\epsilon$ and the residual (or offset) error $C_{\text {res }}$. Noting that $C_{\mathrm{TB}} / C_{R}=2$, we have from (15)

$$
\epsilon \simeq C_{p} / 2 C_{H} \text {. }
$$

The top-plate stray capacitance $C_{p}$ ranges from 0.1 to 1 percent of $C_{H}$, depending on the capacitor size and technology [9]. Therefore, the relative error can be reduced to a value as small as 0.1 percent.

To obtain a rough estimate of the residual capacitance $C_{\text {res }}$, suppose that we are measuring a small capacitance using a 1-pF standard capacitor for $C_{S}$. Assume also the following pessimistic values for the op-amp and circuit parameters: $A_{1}=A_{2}=60 \mathrm{~dB}, V_{\mathrm{os}_{1}}=V_{\mathrm{os}_{2}}=30 \mathrm{mV}, C_{A_{1}}=C_{A_{2}}=1 \mathrm{pF}$, $C_{f}=C_{\epsilon}=C_{\delta}=10 \mathrm{fF},\left(C_{p}+C_{A_{2}}\right) / 2 C_{H}=10^{-3}, V_{R}^{2}=3 \mathrm{~V}$, $V_{\phi} / V_{R}=4$, and $C_{S}<<2 C_{R}$. Then, the residual capacitance due to the clock feedthrough is $40 \mathrm{fF}$, while that due to the offset voltages of the op-amps is about $5 \times 10^{-5} C_{S}$, although its precise value depends on $C_{X}$. This result indicates that the feedthrough component dominates $C_{\text {res }}$, and thus $C_{\text {res }}$ is positive irrespective of the offset voltage polarity.

Consider now the bridge operation in the $Z$ cycle. Since now $Z=1, C_{X}$ is disconnected from the reference source and is grounded. Therefore, the first term in the numerator of (8) vanishes and thus $C_{\text {meas }}$ in (14) equals $C_{\text {res }}$; that is, the bridge in the $Z$ cycle measures the residual capacitance which should be subtracted from the value measured in the $T$ cycle. This self-calibrating ability allows the precise measurement of small capacitances.

Summarizing this section, we can conclude that a 10-bit quantization accuracy and a relative error as small as 0.1 percent is attainable with the integrated version of the bridge.

\section{Measurement Examples}

A prototype bridge has been built using one half of an LM347 quad JFET op-amp chip, MC14066 CMOS analog switches, and discrete capacitors. The capacitor array was controlled by 10 bits. To reduce the capacitance spread in the array, a split reference source was incorporated into the bridge. The source supplied $3.2 \mathrm{~V}$ and $0.1\left(=3.2 / 2^{5}\right) \mathrm{V}$ to two 5-bit arrays, each consisting of 4-, 2-, 1-, 0.5-, and $0.25-\mathrm{nF}$ capacitors. These two arrays were combined so that the array driven by $3.2-\mathrm{V}$ source formed the upper half, and that driven by $0.1-\mathrm{V}$ source the lower half, of the 10-bit array. Another 0.25-nF capacitor was incorporated into the lower half of the array, to form another LSB. Capacitors $C_{R}, C_{H_{1}}$, and $C_{H_{2}}$ were chosen to be $4 \mathrm{nF}$.

Fig. 4 shows the voltage waveforms observable at the noninverting input terminal of op-amp $A_{2}$ during the $B$ state when a ceramic capacitor (nominal value $2 \mathrm{pF}$ ) was measured with reference to a $C_{S}=11.71 \mathrm{pF}$ capacitor. In the figure, the $\phi=1$

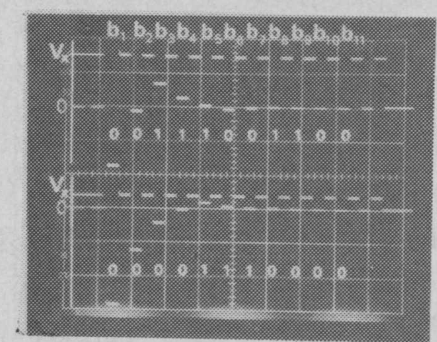

Fig. 4. Waveforms appearing at the noninverting input terminal of opamp $A_{2}$ during the $B$ state, when a $C_{X} \simeq 2 \mathrm{pf}$ ceramic capacitor was measured with reference to a $C_{S}=11.71 \mathrm{pF}$ capacitor. Upper trace: waveform in the $T$ cycle. Lower trace: waveform in the $Z$ cycle. Vertical scale: $0.5 \mathrm{~V} /$ div. Horizontal scale: $0.2 \mathrm{~ms} /$ div.

phase in time slot $T_{i}(i=1,2, \cdots, 11)$ is indicated by $b_{i}$ so that each bit can be identified by the voltage level. Also shown (indicated by $V_{X}$ ) is the voltage stored in $C_{H_{2}}$ in the previous $S$ state. The upper trace shows the waveform in the $T$ cycle. Inspecting the voltage levels ${ }^{1}$ in the $b_{i}$ phases, one obtains the

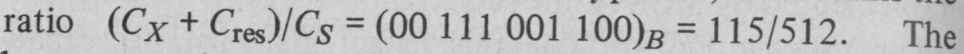
lower trace shows the waveform in the $Z$ cycle. Now one obtains the ratio $C_{\text {res }} / C_{S}=(00001110000)_{B}=7 / 128$. Thus $C_{X}=1.99 \mathrm{pF}$ and $C_{\mathrm{res}}=0.64 \mathrm{pF}$. The measured capacitance of $C_{X}$ agrees exactly with that measured by a commercially available four-terminal-pair bridge. This bridge displays the result in a four-digit BCD form and its measurement error is 0.1 percent +3 LSB. The measured residual capacitance, which is ten times larger than that estimated in the previous section, was found experimentally to be mostly due to the large clock feedthrough of the CMOS analog switch used for the reset transistor $M_{1}$ which is connected to the virtual ground created by op-amp $A_{1}$.

A large number of capacitors were measured, and the results were compared with those obtained by the abovementioned commercial bridge. Capacitors ranging from $10 \mathrm{pF}$ to $1 \mu \mathrm{F}$ in decade steps were used for $C_{S}$, so that $C_{X} / C_{S}$ was less than 1. Table I lists some of the results. It can be seen that the discrepancies between the measured results are mostly within 1 percent, although they tend to increase with decreasing capacitance. This probably can be attributed to the large clock feedthrough of the MOS switches, which violates the assumption that the charges due to paired feedthrough capacitances are negligible. In an LSI realization, these error sources will be greatly reduced.

\section{Conclusions}

An automatic switched-capacitor bridge, which allows the precise measurements of discrete and MOS IC capacitors, has been described. Error analysis has shown that a 10-bit quantization accuracy and a relative error as small as 0.1 percent are attainable by fabricating the bridge using presently available MOS technologies. A prototype bridge, implemented using discrete components, has confirmed the principles of opera-

\footnotetext{
${ }^{1}$ For $b_{7}-b_{11}$, the values are too small to show the sign of the signal. However, they are sufficient to drive the comparator op-amp $A_{2}$ unambiguously.
} 
TABLE I

Comparison between the Capacitances $\mathrm{C}_{\text {Scb }}$ Measured by the Present Switched-Capacitor Bridge and Those $C_{\text {Ftb }}$ By the COMMercial Four-Terminal-Pair Bridge

$\left(C_{n o m}\right.$ denotes the nominal capacitance and $\epsilon_{r}$ is the deviation between $\mathrm{C}_{\mathrm{SCB}}$ and $\mathrm{C}_{\mathrm{FTB}}$ )

\begin{tabular}{|r|c|c|c|}
\hline $\mathrm{C}_{\mathrm{nom}}(\mathrm{pF})$ & $\mathrm{C}_{\mathrm{SCB}}(\mathrm{pF})$ & $\mathrm{C}_{\mathrm{FTB}}(\mathrm{pF})$ & $\varepsilon_{\mathrm{r}}(\%)$ \\
\hline 1 & 1.062 & 1.044 & 1.72 \\
2 & 1.960 & 1.992 & 1.61 \\
4 & 4.324 & 4.227 & 1.10 \\
10 & 10.42 & 10.39 & 0.29 \\
22 & 21.74 & 21.95 & 0.96 \\
47 & 45.48 & 45.42 & 0.13 \\
100 & 103.5 & 102.8 & 0.68 \\
220 & 206.1 & 205.4 & 0.34 \\
470 & 455.8 & 453.3 & 0.55 \\
1000 & 960.5 & 952.4 & 0.85 \\
2200 & 2450 & 2448 & 0.08 \\
4700 & 4528 & 4518 & 0.22 \\
10000 & 10616 & 10530 & 0.82 \\
22000 & 23016 & 23170 & 0.65 \\
40000 & 38695 & 38350 & 0.90 \\
\hline
\end{tabular}

tion. Incorporating a split reference source, a standard capacitor bank, and an automatic range-selecting circuit will provide the bridge with a wide measurement range. The extension to the measurement of grounded capacitors is another future problem.

The bridge described here features a circuit configuration suitable for LSI realization and high accuracy made possible by the digital balance operation and the parasitic-insensitive configuration. Therefore, it is also useful for the interface for "smart" capacitive transducers.

\section{ACKNOWLEDGMENT}

The authors are grateful to K. Kondo of the Research Institute of Electronics, Shizuoka University, for his help in the construction and the measurement of the bridge.

\section{REFERENCES}

[1] B. M. Oliver and J. M. Cage, Electronic Measurements and Instrumentation. New York: McGraw-Hill, 1971, ch. 9.

[2] A. C. Corney, "A universal four-pair impedance bridge," IEEE Trans. Instrum. Meas., vol. IM-28, pp. 211-215, Sept. 1979.

[3] Hewlett Packard Operating Manual: Model 4275A Multi-frequency LCR Meter, Mar. 1982.

[4] J. L. McCreary and D. A. Sealer, "Precision capacitor ratio measurement technique for integrated circuit capacitor arrays," IEEE Trans. Instrum. Meas., vol. IM-28, pp. 11-17, Mar. 1979.

[5] K. Watanabe and G. C. Temes, "Switched-capacitor digital multiplier," Electron. Lett., vol. 19, pp. 33-34, Jan. 20, 1983.

[6] R. Gregorian and S. Fan, "Offset-free high resolution D/A converter," in Proc. 14th Asilomar Conf. on Circuits, Systems, and Computers, Nov. 1980, pp. 316-319.

[7] J. L. McCreary, "Matching properties, and voltage and temperature dependence of MOS capacitors," IEEE J. Solid-State Circuits, vol. SC-16, pp. 608-616, Dec. 1981.

[8] J. L. McCreary and P. R. Gray, "All-MOS charge redistribution analog-to-digital conversion techniques-Part I," IEEE J. SolidState Circuits, vol. SC-10, pp. 371-379, Dec. 1975.

[9] R. W. Brodersen, P. R. Gray, and D. A. Hodges, "MOS switchedcapacitor filters," Proc. IEEE, vol. 67, pp. 61-75, Jan. 1979. 